\title{
A review of transfer learning for medical image classification
}

Hee E. Kim ( $\square$ HeeEun.Kim@medma.uni-heidelberg.de )

Heidelberg University

Alejandro Cosa-Linan

Heidelberg University

Mate E. Maros

Heidelberg University

Nandhini Santhanam

Heidelberg University

Mahboubeh Jannesari

Heidelberg University

Thomas Ganslandt

Heidelberg University

\section{Research Article}

Keywords: imaging, image classification, ResNet

Posted Date: August 26th, 2021

DOl: https://doi.org/10.21203/rs.3.rs-844222/v1

License: (c) (i) This work is licensed under a Creative Commons Attribution 4.0 International License.

Read Full License 


\section{Abstract}

22 This review paper provides an overview of the peer-reviewed articles using transfer learning for

23 medical image analysis, while also providing guidelines for selecting a convolutional neural

24 network model and its configurations for the image classification task. The data characteristics

25 and the trend of models and transfer learning types in the medical domain are additionally

Hee E. Kim,\#, MSc; Alejandro Cosa-Linan¹, PhD; Mate E. Maros ${ }^{1}$, MD, MSc; Nandhini Santhanam ${ }^{1}$, MSc; Mahboubeh Jannesari ${ }^{1}$, MSc; Thomas Ganslandt ${ }^{1}$ MD

${ }^{1}$ Department of Biomedical Informatics at the Center for Preventive Medicine and Digital Health (CPD-BW), Medical Faculty Mannheim, Heidelberg University, Mannheim, Germany

${ }^{\#}$ Corresponding author:

Hee E. Kim, MSc

Department of Biomedical Informatics at the Center for Preventive Medicine and Digital Health (CPD-BW), Medical Faculty Mannheim, Heidelberg University

Theodor-Kutzer-Ufer 1-3, 68167 Mannheim, Germany

Tel.: +49621383 8072

Email: HeeEun.Kim@medma.uni-heidelberg.de

ORCID iD: 0000-0002-2826-2796

Keywords: Transfer learning, Fine-tuning, Deep learning, Convolutional neural network, Medical image analysis

Tables: 2; Figures: 5.

Word count: 3,840

Abstract word count: 214 analyzed. Publications were retrieved from the databases PubMed and Web of Science of peerreviewed articles published in English until December 31, 2020. We followed the PRISMA guidelines for the paper selection and 121 studies were regarded as eligible for the scope of this review. With respect to the model, the majority of studies $(n=57)$ empirically evaluated 
numerous models followed by deep $(n=33)$ and shallow $(n=24)$ models. With respect to the

31 transfer learning approaches, the majority of studies $(n=46)$ empirically searched for the optimal

32 transfer learning configuration followed by feature extractor $(n=38)$ and fine-tuning scratch

$33(n=27)$, feature extractor hybrid $(n=7)$ and fine-tuning $(n=3)$. The investigated studies showed

34 that transfer learning demonstrates either a better or at least a similar performance compared to

35 medical experts despite the limited data sets. We hence encourage data scientists and

36 practitioners to use models such as ResNet or Inception with a feature extractor approach,

37 which saves computational costs and time without degrading the predictive power.

\section{1. Introduction}

39 Medical image analysis (MIA) is a mature research topic, with millions of studies in the field

40 having been published in the last decades. The recent (re-)emergences of deep learning (DL)

41 algorithms and especially convolutional neural networks (CNN) have become the workhorse for

42 MIA. Due to the well-known challenges of MIA, including the small size of medical datasets and

43 the scarcity and cost of high-quality, expert-annotated training data compared to general domain

44 tasks such as ImageNet [1], transfer learning (TL) has emerged as a common practice. Instead

45 of proposing yet novel neural network architecture and training it from scratch, TL utilizes

46 pretrained $\mathrm{CNN}$ models and the knowledge which they learned on natural images to analyze

47 medical images. Although TL has proven to be a valuable tool by improving accuracy,

48 robustness and computational efforts, implementation-focused guidelines for practitioners of this

49 domain are lacking. Therefore, this paper aims to investigate the TL of CNN and to provide

50 insightful recommendations to DL-practitioners on how to leverage TL for MIA by performing a

51 scoping review (of 425 papers) based on the PRISMA guidelines and by summarizing 121

52 papers. 
53 Automated medical image analysis has been applied to support clinicians in various decision-

54 making applications, such as the computer-aided detection of pathologies and disease

55 diagnosis/prognosis. Traditionally, the sophisticated extraction of discriminant handcrafted

56 features so-called feature engineering methodologies such as HOG features or LBP features

57 have dominated the field, but the recent emergence of deep learning algorithms has moved

58 towards non-handcrafted engineering, allowing systems to discover the features that optimally

59 solve the problem at hand. Shi et al. [2] have demonstrated that the features extracted from

60 deep learning surpass the handcrafted method. Convolutional neural networks (CNN) are a

61 special type of deep learning method that processes grid-like topology data such as image data.

62 Unlike the standard neural network consisting of fully connected layers only, CNN consists of at

63 least one convolutional layer. In recent grand challenges organized within the area of medical

64 image analysis, all top-ranked teams have utilized CNN. For instance, the top ten ranked

65 solutions, with the exception of the solution in the ninth rank, have utilized CNN in

66 CAMELYON17 data challenge [3].

67 As of June 12, 2021, 255,000 studies were listed in Google Scholar with the search terms

68 "novel convolutional neural network". This shows that a tremendous amount of research

69 endeavors has been dedicated to designing novel network architectures. The pioneer was

70 LeCun who introduced LeNet in 1998 [4], and consequently, global IT corporations such as

71 Google and Facebook have started to contribute with their architectures and tools in order to

72 democratize the use of deep learning technologies. ResNet [5], GoogLeNet [6], VGG [7] and

73 AlexNet [8] are commonly used CNN models across academia and industries.

74 Liberating this deep knowledge has made the re-use of successfully pretrained models a 75 common practice, rather than developing yet another novel neural network for any new 76 application. This approach is known as transfer learning (TL). TL intends to resemble neural 77 activity as we can learn a new task by adapting prior knowledge learned in similar or different 
78 domains. More specifically, it learns how to analyze medical images from the model learned

79 from natural images. In this way, transfer learning can address one of the most well-known

80 challenges in medical image analysis, namely the small size of the dataset and the scarcity of

81 annotated data compared to general domain tasks such as ImageNet [1], which consists of

82 millions of natural images with over 1000 classes.

83 Litjens et al. [9] have recently published a comprehensive review of the application of deep

84 learning that has been applied to medical images. However, transfer learning is mentioned only

85 briefly. Therefore, this paper aims to investigate transfer learning of CNN and to provide

86 insightful recommendations to readers on optimal architecture choice and hyperparameter

87 setting for optimal leverage of TL with respect to medical image analysis.

\section{2. Background}

89 Transfer learning stems from cognitive research which reproduces the idea that knowledge is

90 transferred across related tasks to improve performance on a new task. It is well-known that

91 humans learn better by retaining and reusing knowledge from solving similar tasks. The formal

92 definition of transfer learning is defined by Pan and Yang with notions of domains and tasks. "A

93 domain consists of a feature space $X$ and a marginal probability distribution $P(X)$, where $X=$

$94\left\{x_{1}, \ldots, x_{n}\right\} \in \mathcal{X}$. Given a specific domain denoted by $\mathcal{D}=\{\mathcal{X}, P(X)\}$, a task consists of a label

95 space $\mathcal{Y}$ and an objective predictive function $f(\cdot)$ denoted by $\mathcal{T}=\{\mathcal{Y}, f(\cdot)\}$. A task is learned

96 from the pair $\left\{x_{i}, y_{i}\right\}$ where $x_{i} \in \mathcal{X}$ and $y_{i} \in \mathcal{Y}$. Given a source domain $\boldsymbol{D}_{\boldsymbol{S}}$ and learning task $\boldsymbol{T}_{\boldsymbol{S}}$,

97 a target domain $\boldsymbol{D}_{T}$ and learning task $\boldsymbol{T}_{\boldsymbol{T}}$, transfer learning aims to help improve the learning of

98 the target predictive function $f_{T}(\cdot)$ in $D_{T}$ by using the knowledge in $\boldsymbol{D}_{\boldsymbol{S}}$ and $\boldsymbol{T}_{\boldsymbol{S}}$, where $\boldsymbol{D}_{\boldsymbol{S}} \neq \boldsymbol{D}_{\boldsymbol{T}}$,

99 or $\boldsymbol{T}_{S} \neq \boldsymbol{T}_{\boldsymbol{T}}$ [10]. 
Analogously, one can learn how to drive a motorbike $\boldsymbol{T}_{\boldsymbol{T}}$ (transferred task) based on its cycling 101 skill $\boldsymbol{T}_{\boldsymbol{s}}$ (source task) where driving two-wheel vehicles can be regarded as the same domain $\boldsymbol{D}_{\boldsymbol{S}}$ $102=D_{T}$. This does not mean that one will not learn how to drive a motorbike without riding a bike.

103 One needs to practice driving the motorbike by adapting one's cycling skills. Similarly, learning 104 the parameters of a network from scratch will require larger annotated datasets and a longer 105 training time to achieve an acceptable performance.

106 The four most common types of transfer learning are visualized in (Fig. 1). Feature extractor 107 and fully connected (FC) layers can be set as non-trainable (frozen) or retrained (trainable) 108 during model fitting. The feature extractor hybrid (Fig. 1a) discards the FC layers and attaches a 109 machine learning algorithm such as SVM or Random Forest classifier into the feature extractor, 110 whereas the skeleton of the given networks remains the same in the other approaches (Fig. 1b$111 \mathrm{~d})$.
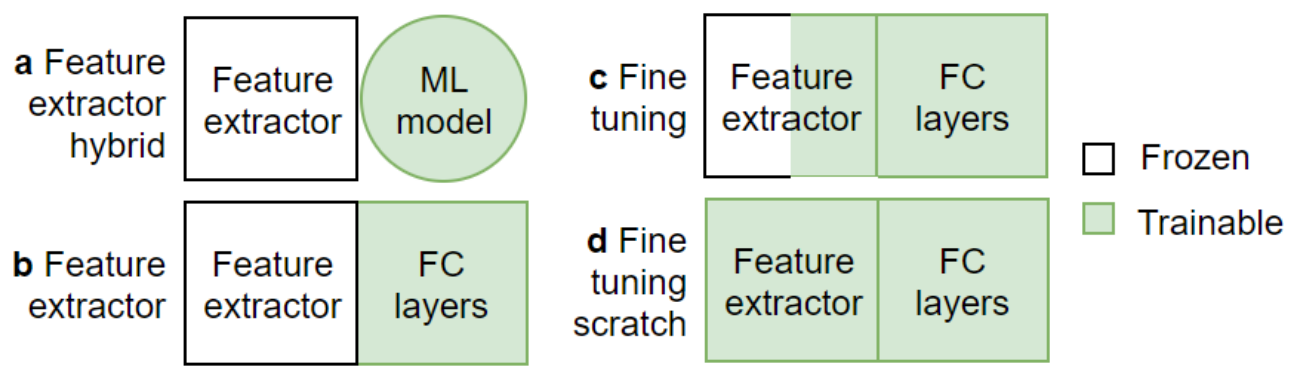

113 Fig. 1 Four types of transfer learning approach

114 Several CNN models are publicly available with pretrained weights and biases. Table 1 115 summarizes the five most popular CNN models for image classification. The number of 116 parameters calculated in one filter is $\left(a{ }^{*} b{ }^{*} c\right)+1$, where $a{ }^{*} b$ is the filter dimension, $c$ is the 117 number of filters in the previous layer and added 1 is the bias. The output of each filter needs to 118 be calculated to obtain the total number of parameters. The models are listed in chronological 119 order from top to bottom. LeNet [4] and AlexNet [8] are the first generations of CNN models 120 developed in 1998 and 2012 respectively. Both are relatively shallow compared to other models 
121 that are developed recently. After AlexNet won the ImageNet challenge in 2012, designing novel

122 networks became an emerging topic among researchers. On the other hand, VGG [7], also

123 referred to as OxfordNet, is recognized as the first deep model. GoogLeNet [11], also known as

124 Inception1, introduced the novel block concept and ResNet introduced residual blocks with skip

125 connections between layers. The inception block employs a set of filters with different sizes and

126 generates the deep networks by concatenating the outputs. However, in the architecture of very

127 deep networks, the weights of the earlier layers are poorly updated during training because they

128 are too far from the output layer. This problem is known as the vanishing gradient problem

129 which is successfully addressed by ResNet by the skip connections. In the classifier head, all

130 models use Softmax function except LeNet-5 which utilizes hyperbolic tangent function. Softmax

131 function fits well with the classification problem because it can convert feature vectors to the 132 probability distribution for each class candidate.

133 Table 1 Overview of five backbone models.

\begin{tabular}{|c|c|c|c|c|c|}
\hline Model & $\begin{array}{c}\text { Released } \\
\text { year }\end{array}$ & $\begin{array}{c}\text { Parameters } \\
\text { (all) }\end{array}$ & $\begin{array}{c}\text { Parameters } \\
\text { (FE only) }\end{array}$ & $\begin{array}{l}\text { Trainable layers } \\
\text { (FE + FC layers) }\end{array}$ & Dataset \\
\hline LeNet5 & 1998 & 60,000 & 1,716 & $4(2+2)$ & MNIST \\
\hline AlexNet & 2012 & $62.3 \mathrm{M}$ & $3.7 \mathrm{M}$ & $8(5+3)$ & \multirow{4}{*}{ ImageNet } \\
\hline VGG16 & 2014 & $134.2 \mathrm{M}$ & $14.7 \mathrm{M}$ & $16(13+3)$ & \\
\hline GoogLeNet & 2014 & $5.3 \mathrm{M}$ & $5.3 \mathrm{M}$ & $22(21+1)$ & \\
\hline ResNet50 & 2015 & $25.6 \mathrm{M}$ & $23.5 \mathrm{M}$ & $51(50+1)$ & \\
\hline \multicolumn{6}{|c|}{$\begin{array}{l}\text { Abbreviations: FE: feature extraction, FC: fully connected layers; MNIST database: Modified National Institute } \\
\text { of Standards and Technology database of handwritten digits with } 60000 \text { training and } 10000 \text { test images, } \\
\text { ImageNet database: organized according to the WordNet hierarchy with over } 14 \text { million hand-annotated } \\
\text { images for visual object recognition research }\end{array}$} \\
\hline
\end{tabular}

\section{3. Methods}

135 Publications were retrieved from two peer-reviewed databases (the PubMed database on

136 January 2, 2021 and the Web of Science database on January 22, 2021). Papers were selected

137 based on the following four conditions: (1) convolutional or CNN should appear in the title or 138 abstract; (2) image data analysis should be considered only; (3) transfer learning models or 
139 pretrained keywords appear in the title or abstract; and finally (4) only experimental studies were

140 considered. The time constraint is specified only for the latest date, which is December 31, 2020.

141 The exact search strings used for these two databases are denoted in Appendix A. Duplicates

142 were merged before screening and eligibility assessment. The first author screened the title,

143 abstract and methods in order to judge whether a study aims to design a novel CNN or not.

144 They were labeled as novel either because a new CNN was proposed and compared to

145 backbone models to prove its efficacy or multiple models including handcrafted features were

146 stacked up. Studies for non-classification and beyond the time coverage were also excluded.

147 For the eligibility assessment, full texts were examined by two researchers. Third independent

148 research was involved in the decision-making in the case that there is any discrepancy between

149 the two researchers.

\section{4. Results}

151 We identified 467 papers after surveying PubMed and Web of Science. (Fig. 2) shows the

152 PRISMA flow diagram of paper selection. After merging 42 duplicates from these two databases,

153425 studies were assessed for screening. 189 studies were excluded because they were either

154 aimed to design a novel CNN or they fell outside the pre-specified time range/published in 2021.

155 In the next step, 236 studies were assessed for eligibility and 114 studies were disqualified from

156 inclusion, resulting in a total of 121 evaluated studies. These selected studies were further

157 investigated and organized with respect to their generic and granular aspects, namely backbone

158 model and transfer learning type. The data characteristics and model performance comparison

159 were also analyzed in order to gain insights regarding the employment of transfer learning in the

160 medical domain. 


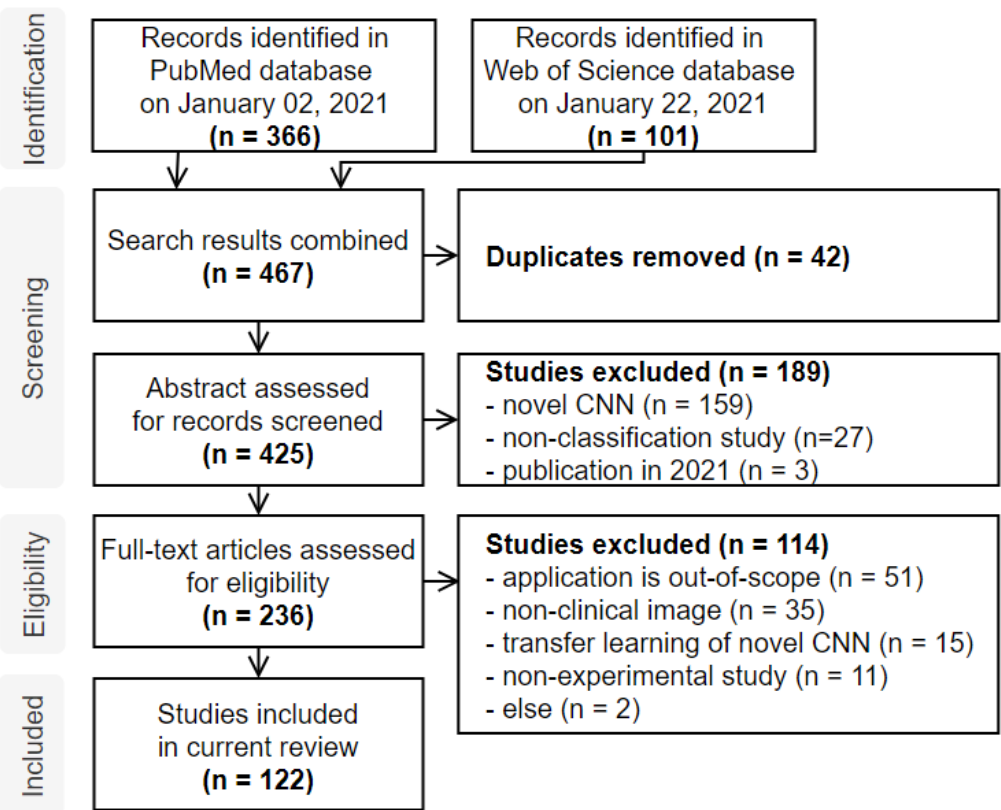

162 Fig. 2 Flowchart of the literature search

163 (Fig. 3a) shows that studies of transfer learning for medical image classification have emerged 164 since 2015 with a 3-year delay after AlexNet [8] won the ImageNet Challenge in 2012. Since 165 then the number of publications grew rapidly for consecutive years. Studies published in 2020 166 seem shrinking compared to the number of publications in 2019 , because the process of 167 indexing a publication may take anywhere from three to six months.
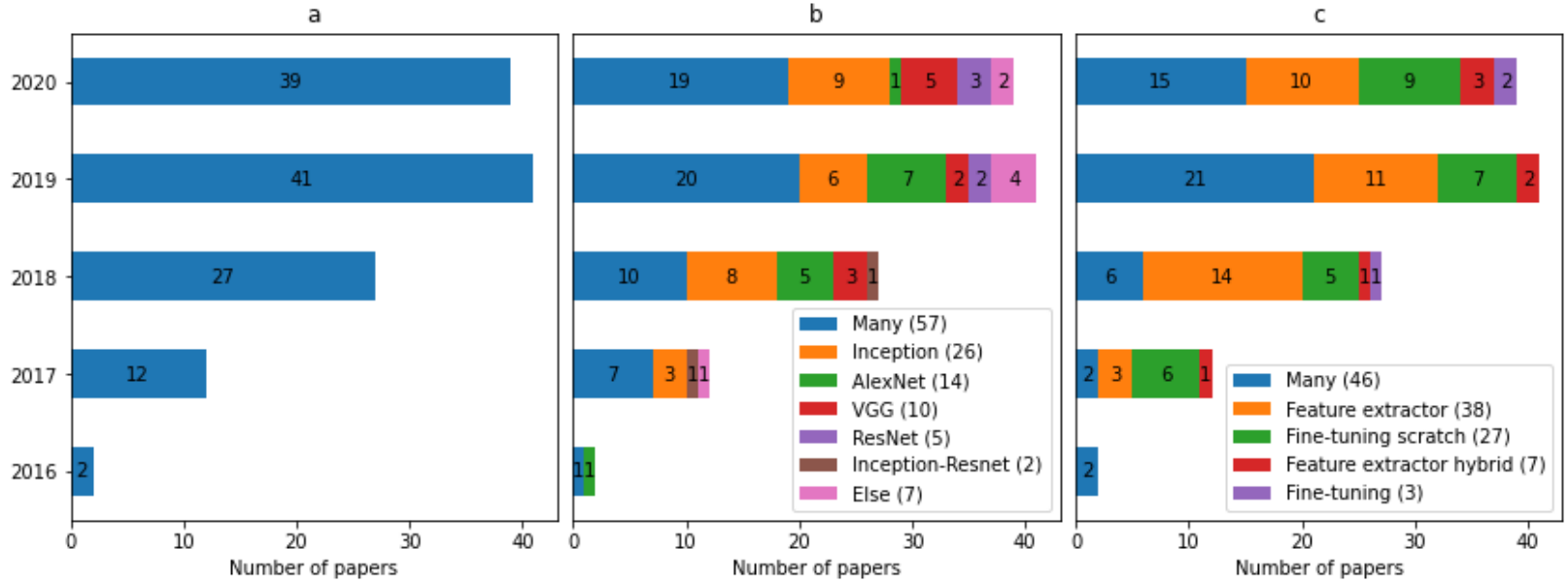

Fig. 3 Studies of transfer learning in medical image analysis over time (y-axis) with respect to a the number of publications, $\mathbf{b}$ applied backbone model and $\mathbf{c}$ transfer learning type 


\section{4.1. Backbone Model}

172 The majority of the investigated studies $(n=57)$ evaluated several backbone models empirically 173 as depicted in (Fig. 3b). Rahaman and his colleagues [12] contributed an intensive benchmark 174 study by evaluating fifteen models, namely: VGG16, VGG19, ResNet50, ResNet101, 175 ResNet152, ResNet50V2, ResNet101V2, ResNet152V2, Inception3, InceptionResNet2, 176 MobileNet1, DenseNet121, DenseNet169, DenseNet201 and XceptionNet. They concluded that 177 VGG19 presented the highest accuracy of $89.3 \%$. This study is exceptional because other 178 studies reported deeper models (Inception and ResNet) which performed better than the 179 shallow models (e.g. VGG and AlexNet). Five studies [13-17] compared Inception and VGG 180 and reported that Inception enjoyed better performance, and Ovalle-Magallanes et al. [18] also 181 concluded that Inception3 performed well compared to ResNet50 and VGG16. Finally, Talo et al. 182 [19] reported that ResNet50 achieved the best classification accuracy compared to AlexNet, 183 VGG16, ResNet18 and ResNet34.

184 Besides the benchmark studies, the most popular model used on its own was the Inception 185 ( $n=26)$ which set the new state of the art in the ImageNet large scale visual recognition 186 challenge (ILSVRC) 2014. The main hallmark was the 1-by-1 filter which transforms a fully 187 connected layer to a convolutional layer, which in turn lowers the computational burden to a 188 great degree.

189 To our surprise, AlexNet $(n=14)$ and VGG $(n=10)$ were the second most used models although 190 they are shallow and early generation models. At first glance, this result seemed counterintuitive 191 because ResNet is a more powerful model with fewer parameters compared to AlexNet and 192 VGG. For instance, ResNet50 achieved a top-5 error of $6.7 \%$ on ILSVRC, which was $2.6 \%$ 193 lower than VGG16 with 5.2 times fewer parameters and 9.7\% lower than AlexNet with 2.4 times 194 fewer parameters [5]. This intuition is valid only if the model was fine-tuned from scratch. The 195 number of parameters drops when the model is utilized as a feature extractor shown in Table 1. 
196 Another assumption was that AlexNet and VGG are easy to understand because the 197 morphology of networks is simply made up of stacked layers. This stands in contrast to more 198 complex concepts such as skip connections, bottlenecks, convolutional blocks introduced in 199 Inception or ResNet.

\section{4.2. Transfer Learning}

201 Similar to the backbone model, the majority of models $(n=46)$ evaluated numerous transfer 202 learning approaches, which are empirically illustrated in (Fig. 3c). Many researchers aimed to 203 search for the optimal choice of the transfer learning approach. Typically, grid search was 204 applied in order to try out as many possible combinations of CNN models with transfer learning 205 approaches.

206 The most popular transfer learning approach was feature extractor $(n=46)$ followed by fine207 tuning scratch $(n=27)$, feature extractor hybrid $(n=7)$ and fine-tuning $(n=3)$. The advantage of 208 feature extractor is that it is able to save computational costs by a large degree compared to the 209 others. Table 2 in Appendix B presents an overview of four transfer learning approaches. 210 Studies are organized based on three dimensions: modality, subject and transfer learning type.

211 Once the chosen model has converged on the new dataset, a part of the model can be fine-

212 tuned. However, we noticed that the fine-tuning ratio was determined arbitrarily and 213 ambiguously. [20] froze all layers except the last 12 layers, while [21, 22] did not clearly 214 describe the fine-tuning configuration but mentioned that it was partially fine-tuned and updated 215 some subsequent layers.

\section{4.3. Data Characteristics}

217 The summary of data characteristics is depicted in (Fig. 4). A variety of human anatomical 218 regions has been studied by means of transfer learning. Most of the studied regions were breast 
cancer exams and skin cancer lesions. Likewise, a wide variety of imaging modalities contained

220 a unique attribute of medical image analysis. For instance, computed tomography (CT) scans

221 and magnetic resonance imaging (MRI) are capable of generating 3D image data, while digital

222 microscopy can generate terabytes of whole slide image (WSI) of tissue specimens. [23]

223 employed native 3D CNN and evaluated multiple CNN models that classify both the 2D

224 mammograms and 3D tomosynthesis. Other studies constructed 2D-CNN or multi-channel CNN

225 with a series of images from 3D input referred to as $2.5 \mathrm{D}-\mathrm{CNN}$, which is able to reduce the

226 processing burden by data sampling without losing model accuracy. (Fig. 4b) shows that the

227 majority of studies consist of binary classes, while (Fig. 4c) shows that the majority of studies

228 have fallen into the first bin which ranges from 0 to 600 . This confirms that most medical studies

229 do not have sufficient data. Even though most hospitals in developed countries archive a large

230 amount of imaging data, researchers often encounter legal issues regarding missing patient

231 consent for scientific analysis. Therefore, only a limited amount of imaging data can be used for

232 analysis. Minor publications are excluded in (Fig. 4) for the following reasons: the experiment

233 was conducted with multiple subjects (human body parts) and/or tasks and/or databases or the

234 subject is non-human body images (e.g. surgical tools).

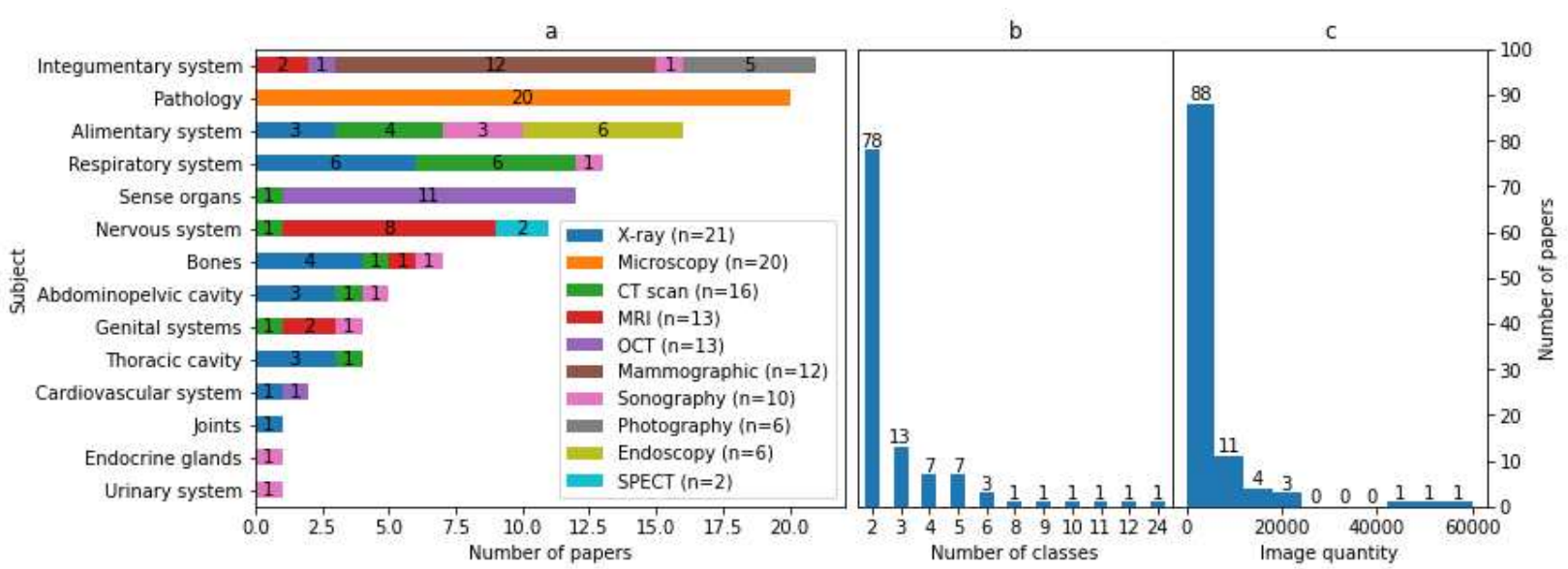

236 Fig. 4 The overview of data characteristics of selected publications. a The correlation of anatomical body parts and imaging modalities. b The number of classes. c The histogram of 


\section{4.4. Performance Visualization}

240 (Fig. 5) shows scatter plots of model performance and transfer learning type and two data 241 characteristics of data size and image modality. The $Y$ coordinates adhere to two metrics, 242 namely area under the receiver operating characteristic curve (AUC) and accuracy. Eleven 243 studies used both metrics, so these are displayed on both scatter plots. The $X$ coordinate is the

244 normalized data quantity otherwise it is not fair to compare the performance of binary 245 classification and classification with ten classes. Note that the data quantities of three modalities 246 - CT, MRI and Microscopy - reflect the number of patients.

247 For the fair comparison, 41 studies employed a single model, transfer learning type, and image 248 modality are depicted. Benchmark studies were excluded, otherwise, one study would generate 249 several overlapping data points and potentially lead to bias. The excluded studies are either with 250 multiple $(n=57)$, with multiple transfer learning types $(n=14)$ or with minor models like LeNet $251(n=9)$.

252 According to Spearman's rank correlation analyses, there were no relevant associations 253 observed between the size of the data set and performance metrics. Data size and AUC (Fig. $2545 a, c)$ showed no relevant correlation $\left(r_{s p}=0.05, p=0.03\right)$. Similarly, only a weak positive trend $255\left(r_{\mathrm{sp}}=0.13, \mathrm{p}=0.17\right)$ could be detected between the size of the dataset and accuracy (Fig. $\left.5 b, d\right)$. 256 There was also no association between other variables such as modality, transfer learning type 257 and backbone model. For instance, the data points of models, such as feature extractors that 258 were fitted onto optical coherence tomography (OCT) images (purple crosses, Fig. 5a,b) 259 showed that larger data quantities did not necessarily guarantee better performance. Notably, 260 data points in cross shapes (models as feature extractors) showed decent results even though 261 only a few fully connected layers were being retrained. 

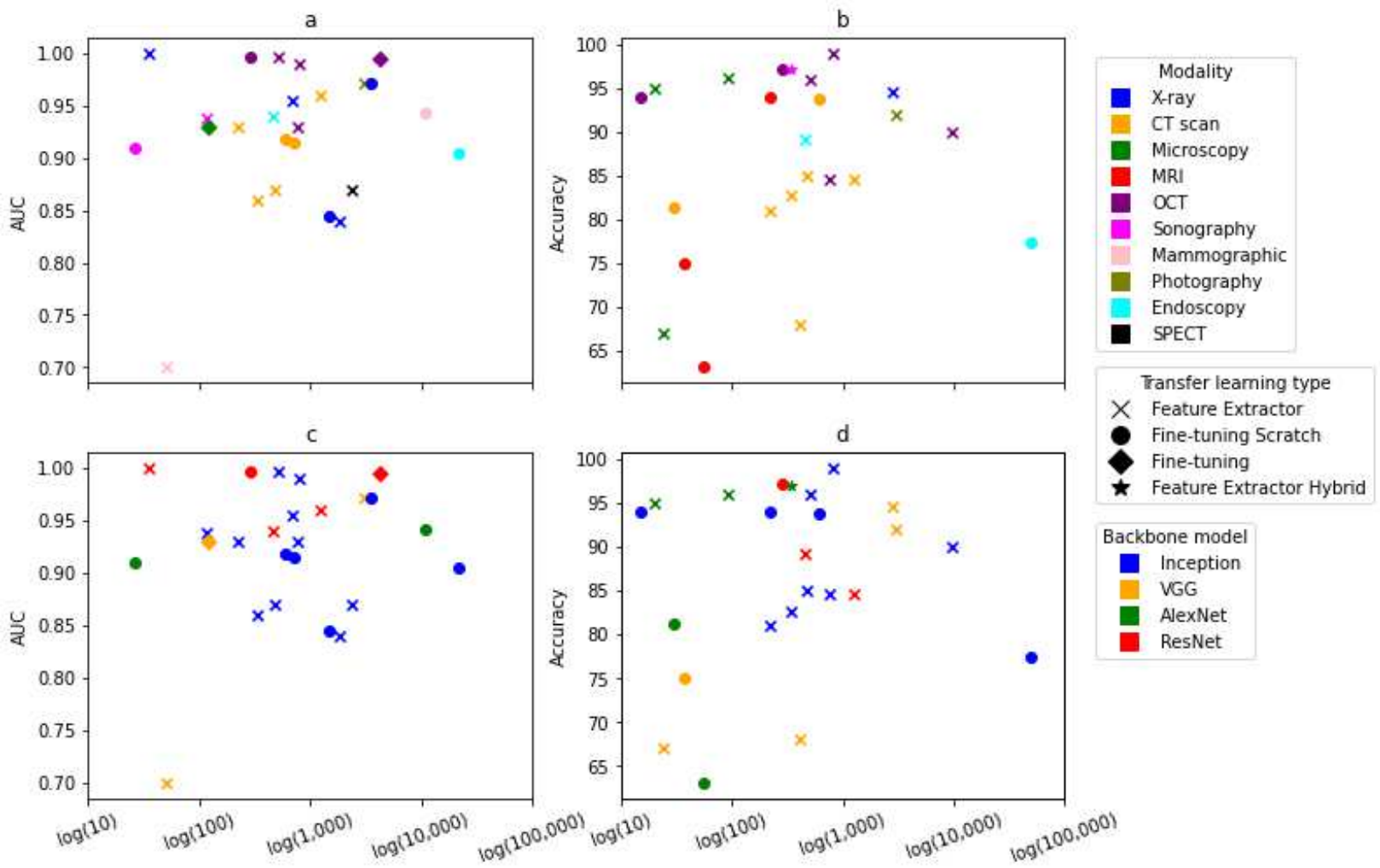

263 Fig. 5 Scatter plots of model performance with data size, image modality and backbone model 264 as well as transfer learning type. Color keys in $\mathbf{a}$ and $\mathbf{b}$ indicate the medical image modality, 265 whereas color keys in $\mathbf{c}$ and $\mathbf{d}$ represent the backbone models. Transfer learning types are in 266 any of four marker shapes for all subfigures.

\section{5. Discussion}

268 We acknowledge that the most used models are Inception, AlexNet, VGG and ResNet, while

269 popular transfer learning configurations are feature extractor and fine-tuning from scratch.

270 However, there is no consensus among studies concerning the global optimum configuration for

271 fine-tuning. [24] concluded that fine-tuning the last fully connected layers of Inception3,

272 ResNet50, and DenseNet121 outperformed fine-tuning from scratch in all cases with AUC

273 values ranging from 0.82 to 0.85 . On the other hand, Yu et al. [25] found that retraining from

274 scratch of DenseNet201 achieved the highest diagnostic accuracy of $92.73 \%$. We speculate

275 that one of the causes is the variety of data subjects and imaging modalities addressed in

276 section 4.4. Therefore, the optimal fine-tuning should be determined empirically, if one takes 
277 into account the fact that, updating all layers (fine-tuning from scratch) might lead to the 278 overfitting to the new dataset, while freezing too many layers may not reflect the characteristics 279 of the new dataset.

280 Fine-tuning from scratch is the second most popular transfer learning approach in the surveyed 281 studies. However, we are discouraged from using this approach for two reasons: firstly, it does 282 not improve the model performance as shown in (Fig. 5) and secondly, it is the most expensive 283 choice because it updates large gradients for entire layers. Therefore, we recommend 284 practitioners to begin with the feature extractor approach.

285 In order to maintain good scientific practice, we encourage practitioners to explore several fine286 tuning configurations such as Lee et al. [26] partitioned VGG16/19 model into 5 blocks, 287 unfreezed blocks sequentially and identified that models that were fine-tuned with two blocks 288 achieved the highest performance. Similarly, [27] fine-tuned the CaffeNet model by unfreezing 289 each layer sequentially. The best results are obtained by the model with one retrained layer for 290 the detection task and with two retrained layers for the classification task. Shin and his 291 colleagues [28] extensively evaluated three components by varying three CNN models (CifarNet, 292 AlexNet and GoogLeNet) with three transfer learning configurations (feature extractor, fine293 tuning from scratch with and without random initialization) and three different image scales 294 (32x32, 64x64 and 256x256). Fine-tuned GoogLeNet from scratch without random initialization 295 was identified as the best performing model.

296 Random initialization often appeared in the literature [29-32]. These studies re-use the skeleton 297 of CNN models only and train the model from scratch. One could argue that there is no transfer 298 of knowledge if the entire weights and biases are initialized, but this is considered as transfer 299 learning in the literature. 
300 This study is limited to survey only the transfer learning for medical image classification. We 301 could not extract a large number of studies for object detection and segmentation, although they 302 have made great progress in various medical applications in the past few years. We assume 303 that the reason for this is because transfer learning is employed as a feature extractor by default

304 for those tasks. For instance, the number of publications studying segmentation or object 305 detection presents at least 100 times more publications in the PubMed database without the 306 keyword of transfer learning.

307 For the future, we expect to see more contributions of specific task oriented transfer learning 308 studies, namely image localization, regression, generative adversarial networks, de-noising, 309 image compression, report generation from images, image synthesis and content-based image 310 retrieval. It is also noteworthy that only few studies employed native 3D-CNN, and the majority 311 of the studies constructed $2 \mathrm{D}-\mathrm{CNN}$ or $2.5 \mathrm{D}-\mathrm{CNN}$ with a sample of images from 3D inputs such 312 as CT and MRI. We expect the number of such studies will be increased in the future as high 313 performance deep learning is an emerging research topic.

314 We also recognize that many studies took advantage of using publicly accessible medical 315 datasets from grand challenges (https://grand-challenge.org/challenges). This is a particularly 316 good practice of science because solutions are shared online for the sake of reproducibility with 317 the dataset. Popular datasets are listed in alphabetical order: BRATS is a dataset that provides 318 MRI scans of the brain for tumor segmentation; CAMELYON contains whole-slide images of 319 hematoxylin and eosin stained lymph node sections; DREAM is a dataset of digital 320 mammography for breast cancer; HAM10000 consists of multi-source dermatoscopic images of 321 pigmented lesions; LUNA16 provides lung cancer screening CT scans for pulmonary nodule 322 detection; PROMISE12 contains MRI scans for prostate segmentation; SLIVER07 is a dataset 323 of CT scans for liver segmentation; and UDIAT, OASBUD and BUSI contain ultrasound images 324 corresponding to breast masses. 


\section{6. Conclusion}

326 Transfer learning has made a large contribution to medical image analysis in the way that it

327 reduces computational costs and mitigates the problem of insufficient data by leveraging

328 knowledge learned on natural images. This paper summarized 121 contributions that employ

329 transfer learning of convolutional neural networks. Our overview of the literature shows that the

330 majority of the studies look for the optimal choice of model and transfer learning setup for the

331 new dataset. Deeper models, such as Inception or ResNet, as a feature extractor are a good

332 starting point. We recommend updating only the last fully connected layers of the chosen model

333 on the medical image dataset. In the case the model performance needs to be refined further,

334 the model could be fine-tuned by incrementally unfreezing convolutional layers with a low 335 learning rate.

\section{Abbreviations}

337 AUC: Area under the receiver operating characteristic curve; CT: Computed tomography; CNN: 338 Convolutional neural networks; DL: Deep learning; FC: Fully connected; ILSVRC: ImageNet 339 large scale visual recognition challenge; MRI: Magnetic resonance imaging; MIA: Medical image 340 analysis; OCT: Optical coherence tomography; TL: Transfer learning; WSL: Whole slide image

\section{Declarations}

\section{Ethics approval and consent to participate}

343 Not applicable.

\section{Consent for publication}

345 Not applicable.

346 Availability of data and materials

347 The dataset analyzed in this study are shown in this appendix B. In depth information is available on 348 reasonable request. 
350 The authors declare that they have no conflict of interest.

$351 \quad$ Funding

352 Not applicable.

\section{3}

354

355

356

\section{2}

\section{Authors' contributions}

HK designed the study, screened the literature review, analyzed the data, the figures and drafted the manuscript. While all authors read and approved the final manuscript, MM and TG critically revised the article. The search query was prepared by HK and AC.

\section{Acknowledgements}

The authors would like to thank Jonathan Griffiths (Heidelberg University and University College, London) and Dr. med. Fabian Siegel (Medical Faculty Mannheim, Heidelberg University) for comments on the manuscript. We would like to thank the reviewer for the constructive feedback.

\section{Appendix A. Search terms}

The search terms used for PubMed were as follows: ("Convolutional neural network*"[Title/Abstract] OR "CNN*"[Title/Abstract]) AND ("image processing, computerassisted"[MeSH Terms] OR "Diagnostic Imaging"[MeSH Terms] OR "medical imag*"[Title/Abstract] OR "clinical imag*"[Title/Abstract] OR "biomedical imag*") AND ("transfer learning"[Title/Abstract] OR "pre-trained"[Title/Abstract] OR "pretrained"[Title/Abstract]) NOT ("Review"[Publication Type] OR "Letter"[Publication Type] OR "meta-analysis"[Publication Type] OR "Systematic Review"[Publication Type] OR "Systematic Review"[Publication Type])

The search string applied in Web of Science database was as follows: TS=("CNN" OR "convolutional") AND TS=("medical imag*" OR "clinical imag*" OR "biomedical imag*") AND TS=("transfer learning" OR "pre-trained" OR "pretrained") NOT TS=("novel" OR "propose")

\section{Appendix B. Supplementary data}

Table 2 A summary table of studies that utilized transfer learning.

\begin{tabular}{|l|l|l|l|}
\hline Modality & Subject & Transfer Learning & Reference \\
\hline CT scan & Abdominopelvic cavity & Feature extractor & {$[33]$} \\
\cline { 2 - 3 } & Alimentary system & Feature extractor & {$[34,35]$} \\
\cline { 3 - 4 } & & Fine-tuning scratch & {$[36,37]$} \\
\hline
\end{tabular}




\begin{tabular}{|c|c|c|c|}
\hline & Bones & Feature extractor & [38] \\
\hline & Genital systems & Fine-tuning scratch & [39] \\
\hline & Nervous system & Many & [40] \\
\hline & Respiratory system & Feature extractor & [41] \\
\hline & & Feature extractor hybrid & [42] \\
\hline & & Fine-tuning scratch & [43-45] \\
\hline & & Many & {$[46,47]$} \\
\hline & Sense organs & Feature extractor & [48] \\
\hline & Thoracic cavity & Feature extractor & [49] \\
\hline Endoscopy & Alimentary system & Feature extractor & {$[50,51]$} \\
\hline & & Fine-tuning scratch & {$[52-54]$} \\
\hline & & Many & [55] \\
\hline Mammographic & Integumentary system & Feature extractor & [2] \\
\hline & & Feature extractor hybrid & {$[56]$} \\
\hline & & Fine-tuning scratch & {$[17,57]$} \\
\hline & & Many & {$[23,25,58-63]$} \\
\hline Microscopy & Pathology & Feature extractor & [64-70] \\
\hline & & Fine-tuning & [21] \\
\hline & & Fine-tuning scratch & {$[71,72]$} \\
\hline MRI & Bones & Many & [73] \\
\hline & Genital systems & Feature extractor & {$[14,74]$} \\
\hline & Integumentary system & Fine-tuning scratch & [75] \\
\hline & & Many & [76] \\
\hline & Nervous system & Fine-tuning scratch & {$[16,77,78]$} \\
\hline & & Many & {$[19,79-81]$} \\
\hline OCT & Integumentary system & Feature extractor & [82] \\
\hline & Cardiovascular system & Many & [83] \\
\hline & Sense organs & Feature extractor & [84-87] \\
\hline & & Feature extractor hybrid & [88] \\
\hline & & Fine-tuning & [20] \\
\hline & & Fine-tuning scratch & {$[29,89]$} \\
\hline & & Many & [90-92] \\
\hline
\end{tabular}




\begin{tabular}{|c|c|c|c|}
\hline \multirow[t]{4}{*}{ Photography } & \multirow[t]{3}{*}{ Integumentary system } & Feature extractor & {$[13,93,94]$} \\
\hline & & Fine-tuning & [22] \\
\hline & & Fine-tuning scratch & [95] \\
\hline & Else & Fine-tuning scratch & [96] \\
\hline \multirow[t]{10}{*}{ Sonography } & Abdominopelvic cavity & Feature extractor & [97] \\
\hline & \multirow[t]{3}{*}{ Alimentary system } & Feature extractor & [98] \\
\hline & & Feature extractor hybrid & [99] \\
\hline & & Fine-tuning scratch & [100] \\
\hline & Bones & Feature extractor & [101] \\
\hline & Endocrine glands & Fine-tuning scratch & [102] \\
\hline & Genital systems & Feature extractor hybrid & [103] \\
\hline & Integumentary system & Many & [104] \\
\hline & Respiratory system & Many & [105] \\
\hline & Urinary system & Feature extractor hybrid & [106] \\
\hline \multirow[t]{2}{*}{ SPECT } & \multirow[t]{2}{*}{ Nervous system } & Feature extractor & [107] \\
\hline & & Many & [108] \\
\hline \multirow[t]{12}{*}{ X-ray } & \multirow[t]{3}{*}{ Abdominopelvic cavity } & Feature extractor & [109] \\
\hline & & Feature extractor hybrid & [110] \\
\hline & & Many & [24] \\
\hline & Alimentary system & Fine-tuning scratch & [111-113] \\
\hline & \multirow[t]{2}{*}{ Bones } & Feature extractor & {$[114,115]$} \\
\hline & & Many & {$[31,116]$} \\
\hline & Cardiovascular system & Many & [117] \\
\hline & Joints & Many & [118] \\
\hline & \multirow[t]{2}{*}{ Respiratory system } & Feature extractor & {$[12,119-121]$} \\
\hline & & Many & {$[26,122]$} \\
\hline & \multirow[t]{2}{*}{ Thoracic cavity } & Fine-tuning scratch & [15] \\
\hline & & Many & {$[32,123]$} \\
\hline Many & Many & Many & {$[28,124]$} \\
\hline
\end{tabular}

\section{$374 \quad$ References}

375 1. ImageNet. http://www.image-net.org/. Accessed 5 Aug 2020. 
2. Shi B, Grimm LJ, Mazurowski MA, Baker JA, Marks JR, King LM, et al. Prediction of Occult Invasive Disease in Ductal Carcinoma in Situ Using Deep Learning Features. J Am Coll Radiol. 2018;15 3 Pt B:527-34.

3. CAMELYON17 - Grand Challenge. grand-challenge.org. https://camelyon17.grandchallenge.org/evaluation/challenge/leaderboard/. Accessed 3 Apr 2021.

4. Lecun Y, Bottou L, Bengio Y, Haffner P. Gradient-based learning applied to document recognition. Proceedings of the IEEE. 1998;86:2278-324.

5. He K, Zhang X, Ren S, Sun J. Deep Residual Learning for Image Recognition. In: 2016 IEEE Conference on Computer Vision and Pattern Recognition (CVPR). Las Vegas, NV, USA: IEEE; 2016. p. 770-8. doi:10.1109/CVPR.2016.90.

6. Szegedy C, Liu W, Jia Y, Sermanet P, Reed S, Anguelov D, et al. Going Deeper with Convolutions. arXiv:14094842 [cs]. 2014. http://arxiv.org/abs/1409.4842. Accessed 30 Jun 2021.

7. Simonyan K, Zisserman A. Very Deep Convolutional Networks for Large-Scale Image Recognition. arXiv:14091556 [cs]. 2015. http://arxiv.org/abs/1409.1556. Accessed 6 Aug 2020. 8. Krizhevsky A, Sutskever I, Hinton GE. ImageNet Classification with Deep Convolutional Neural Networks. In: Pereira F, Burges CJC, Bottou L, Weinberger KQ, editors. Advances in Neural Information Processing Systems 25. Curran Associates, Inc.; 2012. p. 1097-105. http://papers.nips.cc/paper/4824-imagenet-classification-with-deep-convolutional-neuralnetworks.pdf. Accessed 5 Aug 2020.

9. Litjens G, Kooi T, Bejnordi BE, Setio AAA, Ciompi F, Ghafoorian M, et al. A Survey on Deep Learning in Medical Image Analysis. Medical Image Analysis. 2017;42:60-88.

10. Pan SJ, Yang Q. A Survey on Transfer Learning. IEEE Transactions on Knowledge and Data Engineering. 2010;22:1345-59.

11. Hegde RB, Prasad K, Hebbar H, Singh BMK. Feature extraction using traditional image processing and convolutional neural network methods to classify white blood cells: a study. Australas Phys Eng Sci Med. 2019;42:627-38.

12. Rahaman MM, Li C, Yao Y, Kulwa F, Rahman MA, Wang Q, et al. Identification of COVID19 samples from chest $X$-Ray images using deep learning: A comparison of transfer learning approaches. XST. 2020;28:821-39.

13. Burdick J, Marques O, Weinthal J, Furht B. Rethinking Skin Lesion Segmentation in a Convolutional Classifier. J Digit Imaging. 2018;31:435-40.

14. Chen Q, Hu S, Long P, Lu F, Shi Y, Li Y. A Transfer Learning Approach for Malignant Prostate Lesion Detection on Multiparametric MRI. Technol Cancer Res Treat. 2019;18:1533033819858363.

15. Lakhani P. Deep Convolutional Neural Networks for Endotracheal Tube Position and X-ray Image Classification: Challenges and Opportunities. J Digit Imaging. 2017;30:460-8.

16. Yang $\mathrm{H}$, Zhang J, Liu Q, Wang Y. Multimodal MRI-based classification of migraine: using deep learning convolutional neural network. Biomed Eng Online. 2018;17:138.

17. Yu S, Liu L, Wang Z, Dai G, Xie Y. Transferring deep neural networks for the differentiation of mammographic breast lesions. Sci China Technol Sci. 2019;62:441-7.

18. Ovalle-Magallanes E, Avina-Cervantes JG, Cruz-Aceves I, Ruiz-Pinales J. Transfer Learning for Stenosis Detection in X-ray Coronary Angiography. Mathematics. 2020;8:1510. 19. Talo M, Yildirim O, Baloglu UB, Aydin G, Acharya UR. Convolutional neural networks for multi-class brain disease detection using MRI images. Comput Med Imaging Graph. 2019;78:101673.

20. Hemelings R, Elen B, Barbosa-Breda J, Lemmens S, Meire M, Pourjavan S, et al. Accurate prediction of glaucoma from colour fundus images with a convolutional neural network that relies on active and transfer learning. Acta ophthalmologica. 2020;98:e94-100.

21. Valkonen M, Isola J, Ylinen O, Muhonen V, Saxlin A, Tolonen T, et al. Cytokeratinsupervised deep learning for automatic recognition of epithelial cells in breast cancers stained 
for ER, PR, and Ki-67. IEEE transactions on medical imaging. 2019;39:534-42. equivalent and often superior performance to derma tolists in onychomycosis diagnos: equivalent and often superior performance to dermatologists in onychomycosis diagnosis: Automatic construction of onychomycosis datasets by region-based convolutional deep neural network. PloS one. 2018;13:e0191493.

23. Zhang X, Zhang Y, Han EY, Jacobs N, Han Q, Wang X, et al. Classification of whole mammogram and tomosynthesis images using deep convolutional neural networks. IEEE transactions on nanobioscience. 2018;17:237-42.

24. Singh V, Danda V, Gorniak R, Flanders A, Lakhani P. Assessment of critical feeding tube malpositions on radiographs using deep learning. Journal of digital imaging. 2019;32:651-5. 25. Yu X, Zeng N, Liu S, Zhang Y-D. Utilization of DenseNet201 for diagnosis of breast abnormality. Machine Vision and Applications. 2019;30:1135-44. 26. Lee K-S, Kim JY, Jeon E, Choi WS, Kim NH, Lee KY. Evaluation of Scalability and Degree of Fine-Tuning of Deep Convolutional Neural Networks for COVID-19 Screening on Chest X-ray Images Using Explainable Deep-Learning Algorithm. Journal of Personalized Medicine. 2020;10:213.

27. Zhang R, Zheng Y, Mak TWC, Yu R, Wong SH, Lau JY, et al. Automatic detection and classification of colorectal polyps by transferring low-level CNN features from nonmedical domain. IEEE journal of biomedical and health informatics. 2016;21:41-7.

28. Shin H-C, Roth HR, Gao M, Lu L, Xu Z, Nogues I, et al. Deep Convolutional Neural Networks for Computer-Aided Detection: CNN Architectures, Dataset Characteristics and Transfer Learning. IEEE Transactions on Medical Imaging. 2016;35:1285-98. 29. Karri SPK, Chakraborty D, Chatterjee J. Transfer learning based classification of optical coherence tomography images with diabetic macular edema and dry age-related macular degeneration. Biomed Opt Express. 2017;8:579-92.

30. Kim Y-G, Kim S, Cho CE, Song IH, Lee HJ, Ahn S, et al. Effectiveness of transfer learning for enhancing tumor classification with a convolutional neural network on frozen sections. Sci Rep. 2020;10:21899. 31. Lee H, Tajmir S, Lee J, Zissen M, Yeshiwas BA, Alkasab TK, et al. Fully Automated Deep Learning System for Bone Age Assessment. J Digit Imaging. 2017;30:427-41. 32. Tang Y-X, Tang Y-B, Peng Y, Yan K, Bagheri M, Redd BA, et al. Automated abnormality classification of chest radiographs using deep convolutional neural networks. npj Digit Med. 2020;3:70.

33. Huang J, Habib A-R, Mendis D, Chong J, Smith M, Duvnjak M, et al. An artificial intelligence algorithm that differentiates anterior ethmoidal artery location on sinus computed tomography scans. J Laryngol Otol. 2020;134:52-5.

34. Yamada A, Oyama K, Fujita S, Yoshizawa E, Ichinohe F, Komatsu D, et al. Dynamic contrast-enhanced computed tomography diagnosis of primary liver cancers using transfer learning of pretrained convolutional neural networks: Is registration of multiphasic images necessary? Int J CARS. 2019;14:1295-301.

35. Peng J, Kang S, Ning Z, Deng H, Shen J, Xu Y, et al. Residual convolutional neural network for predicting response of transarterial chemoembolization in hepatocellular carcinoma from CT imaging. Eur Radiol. 2020;30:413-24.

36. Hadj Saïd M, Le Roux M-K, Catherine J-H, Lan R. Development of an Artificial Intelligence Model to Identify a Dental Implant from a Radiograph. International Journal of Oral \& Maxillofacial Implants. 2020;35.

37. Lee J-H, Kim D-H, Jeong S-N. Diagnosis of cystic lesions using panoramic and cone beam computed tomographic images based on deep learning neural network. Oral Dis. 2020;26:1528.

38. Parmar P, Habib AR, Mendis D, Daniel A, Duvnjak M, Ho J, et al. An artificial intelligence algorithm that identifies middle turbinate pneumatisation (concha bullosa) on sinus computed 
tomography scans. The Journal of Laryngology \& Otology. 2020;134:328-31. 39. Kajikawa T, Kadoya N, Ito K, Takayama Y, Chiba T, Tomori S, et al. Automated prediction of dosimetric eligibility of patients with prostate cancer undergoing intensity-modulated radiation therapy using a convolutional neural network. Radiological physics and technology. 2018;11:320-7.

40. Dawud AM, Yurtkan K, Oztoprak H. Application of deep learning in neuroradiology: Brain haemorrhage classification using transfer learning. Computational intelligence and neuroscience. 2019;2019.

41. Zhao X, Qi S, Zhang B, Ma H, Qian W, Yao Y, et al. Deep CNN models for pulmonary nodule classification: model modification, model integration, and transfer learning. Journal of $X-$ ray Science and Technology. 2019;27:615-29.

42. da Nobrega RVM, Rebouças Filho PP, Rodrigues MB, da Silva SP, Junior CMD, de Albuquerque VHC. Lung nodule malignancy classification in chest computed tomography images using transfer learning and convolutional neural networks. Neural Computing and Applications. 2018;:1-18.

43. Zhang S, Sun F, Wang N, Zhang C, Yu Q, Zhang M, et al. Computer-aided diagnosis (CAD) of pulmonary nodule of thoracic $\mathrm{CT}$ image using transfer learning. Journal of digital imaging. 2019;32:995-1007.

44. Nibali A, He Z, Wollersheim D. Pulmonary nodule classification with deep residual networks. Int J Comput Assist Radiol Surg. 2017;12:1799-808.

45. Pham TD. A comprehensive study on classification of COVID-19 on computed tomography with pretrained convolutional neural networks. Sci Rep. 2020;10:16942.

46. Xiong J, Li X, Lu L, Schwartz LH, Fu X, Zhao J, et al. Implementation Strategy of a CNN Model Affects the Performance of CT Assessment of EGFR Mutation Status in Lung Cancer Patients. IEEE Access. 2019;7:64583-91.

47. Gao J, Jiang Q, Zhou B, Chen D. Lung Nodule Detection using Convolutional Neural Networks with Transfer Learning on CT Images. Combinatorial Chemistry \& High Throughput Screening. 2020.

48. Chowdhury NI, Smith TL, Chandra RK, Turner JH. Automated classification of osteomeatal complex inflammation on computed tomography using convolutional neural networks. In: International forum of allergy \& rhinology. Wiley Online Library; 2019. p. 46-52. 49. Nishio M, Sugiyama O, Yakami M, Ueno S, Kubo T, Kuroda T, et al. Computer-aided diagnosis of lung nodule classification between benign nodule, primary lung cancer, and metastatic lung cancer at different image size using deep convolutional neural network with transfer learning. PloS one. 2018;13:e0200721.

50. Zachariah R, Samarasena J, Luba D, Duh E, Dao T, Requa J, et al. Prediction of Polyp Pathology Using Convolutional Neural Networks Achieves "Resect and Discard" Thresholds. Am J Gastroenterol. 2020;115:138-44.

51. Zhu Y, Wang Q-C, Xu M-D, Zhang Z, Cheng J, Zhong Y-S, et al. Application of convolutional neural network in the diagnosis of the invasion depth of gastric cancer based on conventional endoscopy. Gastrointestinal Endoscopy. 2019;89:806-815.e1.

52. Cho B-J, Bang CS, Park SW, Yang YJ, Seo SI, Lim H, et al. Automated classification of gastric neoplasms in endoscopic images using a convolutional neural network. Endoscopy. 2019;51:1121-9.

53. Shichijo S, Nomura S, Aoyama K, Nishikawa Y, Miura M, Shinagawa T, et al. Application of Convolutional Neural Networks in the Diagnosis of Helicobacter pylori Infection Based on Endoscopic Images. EBioMedicine. 2017;25:106-11.

54. Shichijo S, Endo Y, Aoyama K, Takeuchi Y, Ozawa T, Takiyama H, et al. Application of convolutional neural networks for evaluating Helicobacter pylori infection status on the basis of endoscopic images. Scand J Gastroenterol. 2019;54:158-63.

55. Patrini I, Ruperti M, Moccia S, Mattos LS, Frontoni E, De Momi E. Transfer learning for 
informative-frame selection in laryngoscopic videos through learned features. Medical \&

56. Samala RK, Chan H-P, Hadjiiski L, Helvie MA. Risks of Feature Leakage and Sample Size Dependencies in Deep Feature Extraction for Breast Mass Classification. Medical Physics. 2020.

57. Mohamed AA, Berg WA, Peng H, Luo Y, Jankowitz RC, Wu S. A deep learning method for classifying mammographic breast density categories. Medical Physics. 2018;45:314-21.

58. Perek S, Kiryati N, Zimmerman-Moreno G, Sklair-Levy M, Konen E, Mayer A. Classification of contrast-enhanced spectral mammography (CESM) images. Int J Comput Assist Radiol Surg. 2019;14:249-57.

59. Samala RK, Chan H-P, Hadjiski L, Helvie MA, Richter CD, Cha KH. Breast cancer diagnosis in digital breast tomosynthesis: effects of training sample size on multi-stage transfer learning using deep neural nets. IEEE transactions on medical imaging. 2018;38:686-96. 60. Huynh BQ, Li H, Giger ML. Digital mammographic tumor classification using transfer learning from deep convolutional neural networks. Journal of Medical Imaging. 2016;3:034501. 61. Chougrad H, Zouaki H, Alheyane O. Deep convolutional neural networks for breast cancer screening. Computer methods and programs in biomedicine. 2018;157:19-30. 62. Samala RK, Chan H-P, Hadjiiski LM, Helvie MA, Richter CD. Generalization error analysis for deep convolutional neural network with transfer learning in breast cancer diagnosis. Physics in Medicine \& Biology. 2020;65:105002.

63. Shen L, Margolies LR, Rothstein JH, Fluder E, McBride R, Sieh W. Deep learning to improve breast cancer detection on screening mammography. Scientific reports. 2019;9:1-12. 64. Shafique S, Tehsin S. Acute Lymphoblastic Leukemia Detection and Classification of Its Subtypes Using Pretrained Deep Convolutional Neural Networks. Technol Cancer Res Treat. 2018;17:1533033818802789.

65. Yu Y, Wang J, Ng CW, Ma Y, Mo S, Fong ELS, et al. Deep learning enables automated scoring of liver fibrosis stages. Scientific Reports. 2018;8:16016.

66. Huttunen MJ, Hassan A, McCloskey CW, Fasih S, Upham J, Vanderhyden BC, et al. Automated classification of multiphoton microscopy images of ovarian tissue using deep learning. Journal of biomedical optics. 2018;23:066002.

67. Talo M. Automated classification of histopathology images using transfer learning. Artificial Intelligence in Medicine. 2019;101:101743.

68. Mazo C, Bernal J, Trujillo M, Alegre E. Transfer learning for classification of cardiovascular tissues in histological images. Computer Methods and Programs in Biomedicine. 2018;165:6976.

69. Riordon J, McCallum C, Sinton D. Deep learning for the classification of human sperm. Computers in biology and medicine. 2019;111:103342.

70. Marsh JN, Matlock MK, Kudose S, Liu T-C, Stappenbeck TS, Gaut JP, et al. Deep learning global glomerulosclerosis in transplant kidney frozen sections. IEEE transactions on medical imaging. 2018;37:2718-28.

71. Kanavati F, Toyokawa G, Momosaki S, Rambeau M, Kozuma Y, Shoji F, et al. Weaklysupervised learning for lung carcinoma classification using deep learning. Sci Rep. 2020;10:9297.

72. Kather JN, Krisam J, Charoentong P, Luedde T, Herpel E, Weis C-A, et al. Predicting survival from colorectal cancer histology slides using deep learning: A retrospective multicenter study. PLOS Medicine. 2019;16:e1002730.

73. He Y, Guo J, Ding X, van Ooijen PM, Zhang Y, Chen A, et al. Convolutional neural network to predict the local recurrence of giant cell tumor of bone after curettage based on pre-surgery magnetic resonance images. European radiology. 2019;29:5441-51.

74. Yuan Y, Qin W, Buyyounouski M, Ibragimov B, Hancock S, Han B, et al. Prostate cancer classification with multiparametric MRI transfer learning model. Medical physics. 2019;46:756- 
65.

75. Borkowski K, Rossi C, Ciritsis A, Marcon M, Hejduk P, Stieb S, et al. Fully automatic classification of breast MRI background parenchymal enhancement using a transfer learning approach. Medicine (Baltimore). 2020;99. doi:10.1097/MD.0000000000021243.

76. Zhu Z, Harowicz M, Zhang J, Saha A, Grimm LJ, Hwang ES, et al. Deep learning analysis of breast MRIs for prediction of occult invasive disease in ductal carcinoma in situ. Computers in biology and medicine. 2019;115:103498.

77. Fukuma R, Yanagisawa T, Kinoshita M, Shinozaki T, Arita H, Kawaguchi A, et al. Prediction of IDH and TERT promoter mutations in low-grade glioma from magnetic resonance images using a convolutional neural network. Scientific reports. 2019;9:1-8.

78. Banzato T, Causin F, Della Puppa A, Cester G, Mazzai L, Zotti A. Accuracy of deep learning to differentiate the histopathological grading of meningiomas on MR images: A preliminary study. Journal of Magnetic Resonance Imaging. 2019;50:1152-9.

79. Swati ZNK, Zhao Q, Kabir M, Ali F, Ali Z, Ahmed S, et al. Brain tumor classification for MR images using transfer learning and fine-tuning. Computerized Medical Imaging and Graphics. 2019;75:34-46.

80. Yang Y, Yan L-F, Zhang X, Han Y, Nan H-Y, Hu Y-C, et al. Glioma grading on conventional MR images: a deep learning study with transfer learning. Frontiers in neuroscience. 2018;12:804.

81. Deepak S, Ameer PM. Brain tumor classification using deep CNN features via transfer learning. Computers in biology and medicine. 2019;111:103345.

82. Singla N, Dubey K, Srivastava V. Automated assessment of breast cancer margin in optical coherence tomography images via pretrained convolutional neural network. Journal of biophotonics. 2019;12:e201800255.

83. Gessert N, Lutz M, Heyder M, Latus S, Leistner DM, Abdelwahed YS, et al. Automatic plaque detection in IVOCT pullbacks using convolutional neural networks. IEEE transactions on medical imaging. 2018;38:426-34.

84. Ahn JM, Kim S, Ahn K-S, Cho S-H, Lee KB, Kim US. A deep learning model for the detection of both advanced and early glaucoma using fundus photography. PloS one. 2018;13:e0207982.

85. Treder M, Lauermann JL, Eter N. Automated detection of exudative age-related macular degeneration in spectral domain optical coherence tomography using deep learning. Graefe's Archive for Clinical and Experimental Ophthalmology. 2018;256:259-65. 86. Zheng C, Xie X, Huang L, Chen B, Yang J, Lu J, et al. Detecting glaucoma based on spectral domain optical coherence tomography imaging of peripapillary retinal nerve fiber layer: a comparison study between hand-crafted features and deep learning model. Graefe's Archive for Clinical and Experimental Ophthalmology. 2020;258:577-85.

87. Zago GT, Andreão RV, Dorizzi B, Salles EOT. Retinal image quality assessment using deep learning. Computers in biology and medicine. 2018;103:64-70.

88. Burlina P, Pacheco KD, Joshi N, Freund DE, Bressler NM. Comparing humans and deep learning performance for grading AMD: a study in using universal deep features and transfer learning for automated AMD analysis. Computers in biology and medicine. 2017;82:80-6. 89. Liu TA, Ting DS, Paul HY, Wei J, Zhu H, Subramanian PS, et al. Deep learning and transfer learning for optic disc laterality detection: Implications for machine learning in neuroophthalmology. Journal of Neuro-Ophthalmology. 2020;40:178-84.

90. Choi JY, Yoo TK, Seo JG, Kwak J, Um TT, Rim TH. Multi-categorical deep learning neural network to classify retinal images: A pilot study employing small database. PloS one. 2017;12:e0187336.

91. Gómez-Valverde JJ, Antón A, Fatti G, Liefers B, Herranz A, Santos A, et al. Automatic glaucoma classification using color fundus images based on convolutional neural networks and transfer learning. Biomedical optics express. 2019;10:892-913. 
631

632

633

634

635

636

637

638

639

640

641

642

643

644

645

646

647

648

649

650

651

652

653

654

655

656

657

658

659

660

661

662

663

664

665

666

667

668

669

670

671

672

673

674

675

676

677

678

679

680

681

92. Xu BY, Chiang M, Chaudhary S, Kulkarni S, Pardeshi AA, Varma R. Deep learning classifiers for automated detection of gonioscopic angle closure based on anterior segment OCT images. American journal of ophthalmology. 2019;208:273-80.

93. Shen X, Zhang J, Yan C, Zhou H. An automatic diagnosis method of facial acne vulgaris based on convolutional neural network. Scientific reports. 2018;8:1-10.

94. Cirillo MD, Mirdell R, Sjöberg F, Pham TD. Time-independent prediction of burn depth using deep convolutional neural networks. Journal of Burn Care \& Research. 2019;40:857-63. 95. Huang K, He X, Jin Z, Wu L, Zhao X, Wu Z, et al. Assistant Diagnosis of Basal Cell Carcinoma and Seborrheic Keratosis in Chinese Population Using Convolutional Neural Network. Journal of healthcare engineering. 2020;2020.

96. Sun Y, Shan C, Tan T, Tong T, Wang W, Pourtaherian A. Detecting discomfort in infants through facial expressions. Physiological measurement. 2019;40:115006.

97. Cheng PM, Malhi HS. Transfer Learning with Convolutional Neural Networks for Classification of Abdominal Ultrasound Images. J Digit Imaging. 2017;30:234-43.

98. Xue L-Y, Jiang Z-Y, Fu T-T, Wang Q-M, Zhu Y-L, Dai M, et al. Transfer learning radiomics based on multimodal ultrasound imaging for staging liver fibrosis. European radiology. 2020;:111.

99. Byra M, Styczynski G, Szmigielski C, Kalinowski P, Michallowski Lukasz, Paluszkiewicz R, et al. Transfer learning with deep convolutional neural network for liver steatosis assessment in ultrasound images. International journal of computer assisted radiology and surgery.

2018;13:1895-903.

100. Banzato T, Bonsembiante F, Aresu L, Gelain ME, Burti S, Zotti A. Use of transfer learning to detect diffuse degenerative hepatic diseases from ultrasound images in dogs: a methodological study. The Veterinary Journal. 2018;233:35-40.

101. Hetherington J, Lessoway V, Gunka V, Abolmaesumi P, Rohling R. SLIDE: automatic spine level identification system using a deep convolutional neural network. Int J CARS.

2017;12:1189-98.

102. Chi J, Walia E, Babyn P, Wang J, Groot G, Eramian M. Thyroid nodule classification in ultrasound images by fine-tuning deep convolutional neural network. Journal of digital imaging. 2017;30:477-86.

103. Sridar P, Kumar A, Quinton A, Nanan R, Kim J, Krishnakumar R. Decision Fusion-Based Fetal Ultrasound Image Plane Classification Using Convolutional Neural Networks. Ultrasound in Medicine \& Biology. 2019;45:1259-73.

104. Byra M, Galperin M, Ojeda-Fournier H, Olson L, O'Boyle M, Comstock C, et al. Breast mass classification in sonography with transfer learning using a deep convolutional neural network and color conversion. Medical physics. 2019;46:746-55.

105. Chen C-H, Lee Y-W, Huang Y-S, Lan W-R, Chang R-F, Tu C-Y, et al. Computer-aided diagnosis of endobronchial ultrasound images using convolutional neural network. Computer methods and programs in biomedicine. 2019;177:175-82.

106. Zheng Q, Furth SL, Tasian GE, Fan Y. Computer-aided diagnosis of congenital abnormalities of the kidney and urinary tract in children based on ultrasound imaging data by integrating texture image features and deep transfer learning image features. Journal of pediatric urology. 2019;15:75-e1.

107. Kim DH, Wit H, Thurston M. Artificial intelligence in the diagnosis of Parkinson's disease from ioflupane-123 single-photon emission computed tomography dopamine transporter scans using transfer learning. Nuclear medicine communications. 2018;39:887-93.

108. Papathanasiou ND, Spyridonidis T, Apostolopoulos DJ. Automatic characterization of myocardial perfusion imaging polar maps employing deep learning and data augmentation. Hellenic Journal of Nuclear Medicine. 2020;23:125-32.

109. Cheng PM, Tejura TK, Tran KN, Whang G. Detection of high-grade small bowel obstruction on conventional radiography with convolutional neural networks. Abdom Radiol. 
2018;43:1120-7.

110. Devnath L, Luo S, Summons P, Wang D. Automated detection of pneumoconiosis with multilevel deep features learned from chest X-Ray radiographs. Comput Biol Med. 2021;129:104125.

111. Kim J-E, Nam N-E, Shim J-S, Jung Y-H, Cho B-H, Hwang JJ. Transfer learning via deep neural networks for implant fixture system classification using periapical radiographs. Journal of clinical medicine. 2020;9:1117. 112. Lee J-H, Kim D-H, Jeong S-N, Choi S-H. Detection and diagnosis of dental caries using a deep learning-based convolutional neural network algorithm. Journal of dentistry. 2018;77:10611.

692 113. Lee J-H, Jeong S-N. Efficacy of deep convolutional neural network algorithm for the identification and classification of dental implant systems, using panoramic and periapical radiographs: A pilot study. Medicine. 2020;99.

695 114. Paul HY, Kim TK, Wei J, Shin J, Hui FK, Sair HI, et al. Automated semantic labeling of pediatric musculoskeletal radiographs using deep learning. Pediatric radiology. 2019;49:106670.

698 115. Kim DH, MacKinnon T. Artificial intelligence in fracture detection: transfer learning from 699 deep convolutional neural networks. Clinical radiology. 2018;73:439-45. 116. Cheng C-T, Ho T-Y, Lee T-Y, Chang C-C, Chou C-C, Chen C-C, et al. Application of a deep learning algorithm for detection and visualization of hip fractures on plain pelvic radiographs. European radiology. 2019;29:5469-77.

117. Ovalle-Magallanes E, Avina-Cervantes JG, Cruz-Aceves I, Ruiz-Pinales J. Transfer Learning for Stenosis Detection in X-ray Coronary Angiography. Mathematics. 2020;8:1510. 118. Abidin AZ, Deng B, DSouza AM, Nagarajan MB, Coan P, Wismüller A. Deep transfer learning for characterizing chondrocyte patterns in phase contrast X-Ray computed tomography images of the human patellar cartilage. Computers in Biology and Medicine. 2018;95:24-33. 119. Heidari M, Mirniaharikandehei S, Khuzani AZ, Danala G, Qiu Y, Zheng B. Improving the performance of CNN to predict the likelihood of COVID-19 using chest X-ray images with preprocessing algorithms. International journal of medical informatics. 2020;144:104284. 120. Albahli S, Albattah W. Deep Transfer Learning for COVID-19 Prediction: Case Study for Limited Data Problems. Current medical imaging. 2020.

121. Minaee S, Kafieh R, Sonka M, Yazdani S, Jamalipour Soufi G. Deep-COVID: Predicting COVID-19 from chest X-ray images using deep transfer learning. Med Image Anal. 2020;65:101794.

122. Apostolopoulos ID, Mpesiana TA. Covid-19: automatic detection from $x$-ray images utilizing transfer learning with convolutional neural networks. Physical and Engineering Sciences in Medicine. 2020;43:635-40.

123. Romero M, Interian $Y$, Solberg $T$, Valdes G. Targeted transfer learning to improve performance in small medical physics datasets. Medical Physics. 2020;47:6246-56. 124. Clancy K, Aboutalib S, Mohamed A, Sumkin J, Wu S. Deep Learning Pre-training Strategy for Mammogram Image Classification: an Evaluation Study. J Digit Imaging. 2020;33:1257-65. 